\title{
Deriving Code Coverage Information from Profiling Data Recorded for a Trace-based Just-in-time Compiler
}

\author{
Christian Häubl \\ Institute for System Software \\ Johannes Kepler University Linz \\ Austria \\ haeubl@ssw.jku.at
}

\author{
Christian Wimmer \\ Oracle Labs \\ USA \\ christian.wimmer@oracle.com
}

\author{
Hanspeter Mössenböck \\ Institute for System Software \\ Johannes Kepler University Linz \\ Austria \\ moessenboeck@ssw.jku.at
}

\begin{abstract}
Code coverage information is typically recorded by adding instrumentation to an application before or during execution. However, this has the disadvantage that the instrumentation decreases the performance.

A high-performance virtual machine (VM), such as Oracle's Java HotSpot VM, is already designed to record profiling data for the executed application. This profiling data is intended to guide optimizations during just-in-time (JIT) compilation. In this paper, we propose a runtime system that allows deriving exact code coverage information from this recorded profiling data by guaranteeing certain system properties. With this approach, we avoid the hassle of instrumenting the application explicitly. Therefore, we minimize both the run-time overhead and the implementation effort for recording code coverage information.

We implemented our approach for a variant of the Java HotSpot VM that uses a trace-based JIT compiler. However, our approach is simple and general enough to be applicable to most modern VMs. Our evaluation with industry-strength benchmark suites shows that this approach allows recording code coverage information while retaining almost full peak performance. So, our approach is also suitable for collecting code coverage information in day-to-day operation environments such as long running server applications.
\end{abstract}

Categories and Subject Descriptors D.2.5 [Software Engineering]: Testing and Debugging_-Testing tools (e.g., data generators, coverage testing); D.3.4 [Programming Languages]: ProcessorsCompilers

General Terms Measurement, Performance

Keywords Java, code coverage, just-in-time compilation

\section{Introduction}

Code coverage metrics are used to determine the statements, branches or paths that were covered during the execution of a pro-

Permission to make digital or hard copies of part or all of this work for personal or classroom use is granted without fee provided that copies are not made or distributed for profit or commercial advantage and that copies bear this notice and the full citation on the first page. Copyrights for third-party components of this work must be honored. For all other uses, contact the owner/author(s).

PPPJ'13, September 11-13, 2013, Stuttgart, Germany.

Copyright is held by the owner/author(s).

ACM 978-1-4503-2111-2/13/09.

http://dx.doi.org/10.1145/2500828.2500829 gram. These metrics are useful for finding out whether the program was tested extensively enough and are usually obtained by instrumenting the code.

Figure 1 shows the steps that are typically involved to record code coverage information for a Java application. The first step is to instrument the application either in an offline preprocessing step or online while the application is executed. The code coverage tool inserts probes at all relevant positions of the control flow. When a probe is executed, it marks a certain part of the application as covered. When the application exits, the coverage data is persisted, either by storing it in a file or transmitting it to another machine. The actual computation of the covered code parts is then done offline by analyzing the recorded coverage data and combining this information with the application's source code.

The problem with this approach is that instrumentation degrades the performance of the executed program and is therefore typically only enabled during testing and switched off in daily operation.

For Java, several code coverage tools exist and to the best of our knowledge, all of them add instrumentation code to the application or to the virtual machine (VM). However, instrumenting an application has some disadvantages such as slowing down the execution or even introducing bugs that cannot be reproduced without instrumentation. Approaches to reduce the overhead of the code coverage probes can be divided into two categories (see Section 5). The first category of optimizations tries to avoid redundant probes so that fewer probes have to be inserted and less instrumentation code has to be executed at run time. The second category removes code coverage probes when they are no longer needed. Removing no longer required probes is efficient but increases the complexity of the code coverage tools.

We propose a system where no instrumentation code has to be added explicitly to record exact code coverage information. Instead, we exploit the fact that modern high-performance VMs already have an instrumented interpreter or baseline compiler that records profiling data. Normally, this profiling data is only used

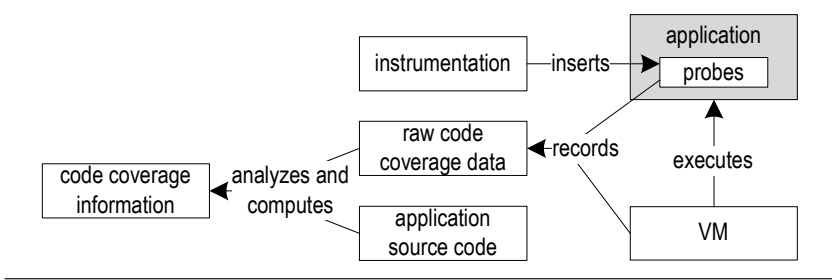

Figure 1. Obtaining code coverage information 


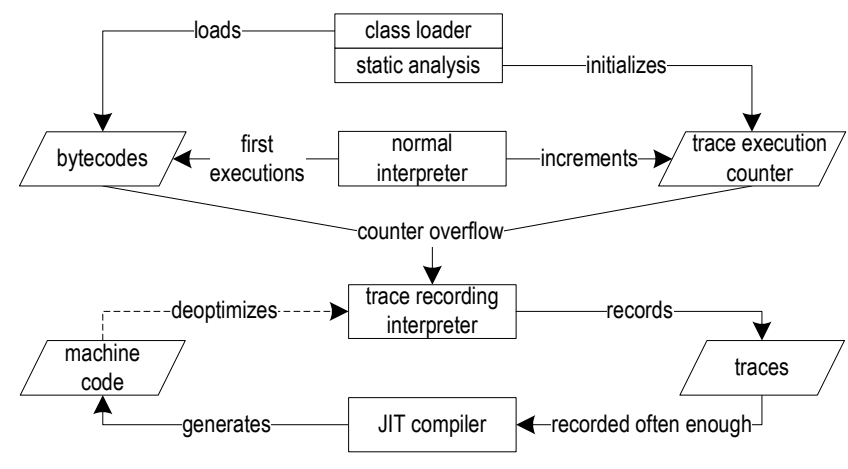

Figure 2. Structure of the tracing VM

during just-in-time (JIT) compilation to guide aggressive and optimistic optimizations such as type specialization and removal of never executed code. However, by guaranteeing certain system properties, this information can be also used to derive exact code coverage information. This allows minimizing the implementation effort to obtain code coverage information, while ensuring that the impact on peak performance is minimal.

Our paper contributes the following:

- We propose a runtime system that can derive accurate code coverage information from existing instrumentation that was originally intended for recording profiling data. This technique is applicable to all modern VMs that record profiling data and have an aggressive JIT compiler. Our system has very low impact on peak performance.

- We compare our approach to other commonly used code coverage approaches and discuss its advantages and drawbacks.

- We evaluate our system on the industry-strength benchmark suites SPECjvm2008 [30] and DaCapo 9.12 Bach [10] in terms of startup performance, peak performance, amount of generated machine code, and time required for JIT compilation. We also compared our approach to a state-of-the-art code coverage tool for Java that uses bytecode instrumentation.

\section{Trace-based HotSpot VM}

We build on a trace recording infrastructure and a trace-based JIT compiler for Java [17-19] that is integrated into Oracle's HotSpot VM. Figure 2 shows the structure of this VM.

Before a Java application starts execution, the class loader loads, parses, and verifies the class files. This class loading step creates run-time data structures such as the constant pool and method objects.

A trace is a sequence of instructions that starts at a so-called trace anchor. The current implementation supports traces that start at method entries or at loop headers. To detect possible loop trace anchors, a bytecode preprocessing step is performed after class loading.

Initial execution of a program starts in the normal interpreter. Whenever a trace anchor is encountered, a counter for this anchor is incremented. When the counter overflows, the specific trace anchor is marked as hot and execution switches to the instrumented trace recording interpreter, which records traces starting at this anchor (see Section 2.1). When enough traces have been recorded they are sent to the JIT compiler (see Section 2.2) and compiled to machine code. On certain occasions, compiled traces can fall back to the trace recording interpreter.

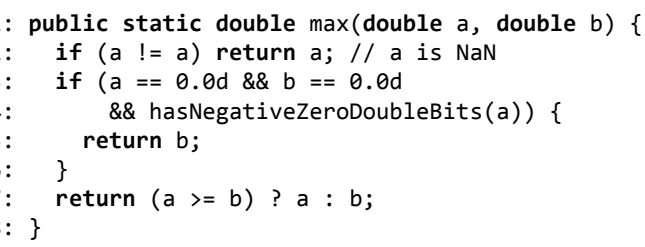

(a) source code

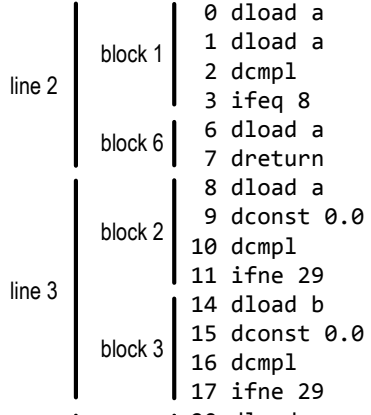

$$
\begin{aligned}
& \text { line 4 } \mid \begin{array}{l|l}
\text { block 4 } \mid \begin{array}{l}
20 \text { dload a } \\
21 \text { invokestatic } \\
\text { hasNegativeZeroDoubleBits() } \\
24 \text { ifeq } 29
\end{array}
\end{array} \\
& \text { line } 5 \mid \text { block } 5 \mid \begin{array}{ll}
27 \text { dload b } \\
28 \text { dreturn }
\end{array}
\end{aligned}
$$

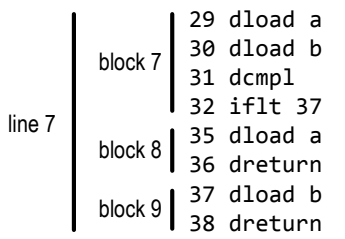

(b) bytecode

Figure 3. Source- and bytecode for the method Math. $\max ()$

\subsection{Trace Recording}

Unlike other trace recording approaches, we restrict traces to span at most one method [17], while traces of invoked methods are linked to the calling trace. Every thread holds a thread-local stack of those traces that are currently being recorded. When the trace recording interpreter encounters a bytecode that modifies the control flow, it records information in the topmost trace of the tracing stack.

When another trace anchor (a method entry or a loop header) is reached during trace recording, a new trace is started and pushed on the tracing stack so that trace recording for this trace anchor continues there. When the execution leaves the trace anchor's method or loop, the corresponding trace is popped from the tracing stack and stored in a trace repository. Then, we link the caller and the callee trace by storing a pointer to the callee's trace in the caller's trace and continue recording for the caller. The linking preserves context-sensitive call information over method boundaries and results in a data structure that is similar to a dynamic call graph.

Figure 3 shows the source- and the bytecode for the method Math $\max ()$, while Figure 4 shows the raw traces that hold the bytecode-level control flow for this method.

Before storing a recorded trace, we check if the same trace was already recorded before. For this, we consider traces to be different if they took a different path or if they invoked different callee traces. If the trace was already recorded, we only increment a counter in the already stored trace. By considering traces as different if they invoke different traces, we are able to record exact call information 


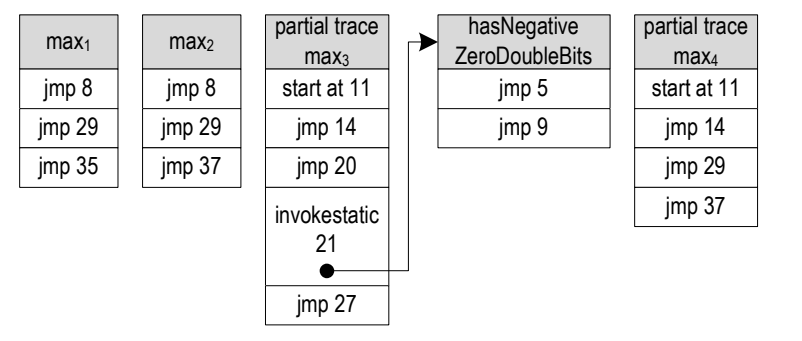

Figure 4. Detailed traces for the method Math.max ()

for every executed path through the whole application. To reduce the number of recorded traces to a reasonable amount, we do not link loop traces and recursive method traces to their parent trace.

\subsection{Trace-based Compilation}

When traces have been recorded often enough for a specific trace anchor, they are passed to the trace-based JIT compiler. The first compilation step merges the recorded traces and generates the compiler's high-level intermediate representation (HIR). Figure 5 (a) shows the control flow graph that would be used in a method-based compiler as the HIR for the method Math. $\max ($ ). Initially, the trace recording interpreter records the traces shown in Figure 5 (b) and merges them into the trace graph [17] shown in Figure 5 (c). On this trace graph, general and tracing-specific optimizations are applied and the optimized trace graph is translated to the low-level intermediate representation (LIR). Then, the LIR is used for linear scan register allocation [32] and code generation. In subsequent executions, the generated machine code is then invoked by one of the interpreters or by other compiled traces. In terms of startup and peak performance, previous work [19] showed that the trace-based compiler can compete with the method-based HotSpot JIT compilers.

During JIT compilation, our trace-based compiler can use aggressive, optimistic optimizations that are guarded by runtime checks. If such a guard is violated during execution or if code must be executed that was not compiled, our system deoptimizes [21] to the trace recording interpreter. The trace recording interpreter continues execution at the point of deoptimization and records a so-called partial trace that starts directly at the point of deoptimization instead of at the trace anchor, as the traces $\max _{3}$ and $\max _{4}$ show in Figure 4 and Figure 5 (d). If a specific piece of generated machine code deoptimizes too frequently, this machine code is invalidated and a new compilation is triggered. For this compilation, both the originally recorded traces and the new partial traces are merged, which results in our example in the trace graph shown in Figure 5 (e). This trace graph now covers all relevant parts of the method so that no further deoptimization is necessary. So, partial traces increase method coverage or disable specific aggressive optimizations, which in turn reduces the deoptimization frequency.

\section{Code Coverage from Trace Information}

For deriving exact code coverage information from the recorded traces, we add certain requirements to our runtime system. This allows obtaining the code coverage information while minimizing the impact on peak performance.

\subsection{Runtime System and Requirements}

Figure 6 shows our modified runtime system that we use to derive accurate code coverage information from the traces recorded for JIT compilation.

Unlike our original trace-based runtime system, the threshold for trace recording is set to zero so that the trace recording inter-

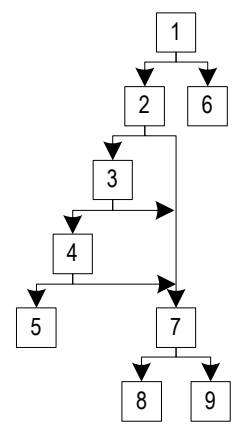

(a) control flow graph

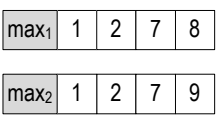

(b) traces

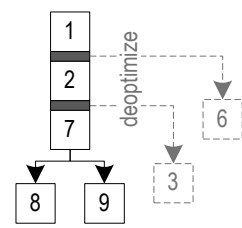

(c) initial trace graph

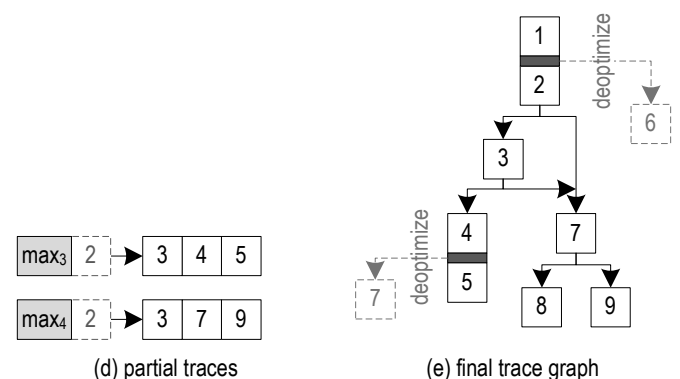

Figure 5. Evolution of the trace graph for the method Math. $\max ()$

preter is used from the beginning. While this has a negative impact on startup performance, it ensures that every executed code is traced. Unexecuted code is not traced and therefore not compiled. In other words, we can be sure that all compiled code has been executed and traced before. When compiled code branches off to uncompiled code, execution falls back to the trace recording interpreter that starts recording a new partial trace. Therefore, the recorded traces always comprise those parts of the code that were executed. So, code coverage information can be collected during trace recording and the compiled code does not have to be instrumented. This avoids any negative impact on the peak performance.

Our approach is not only applicable to runtime systems with trace-based JIT compilers, but can be generalized to any aggressive JIT compiler that uses profiling data to guide its optimizations. It is only necessary to fulfill the following requirements and guarantees:

- The VM must use an interpreter or a baseline compiler that is instrumented for recording profiling data. In particular, it is necessary that the recorded profiling data indicates which code parts have been executed. To obtain edge coverage information, the recorded profiling data must also store which control flow edges have been executed. One way to do that is to store the taken and not taken counts for branches.

- Profiling data must be recorded whenever uncompiled code is executed. Furthermore, the JIT compiler must only compile code that has been executed. For edge coverage, it is also required that the JIT compiler only compiles control flow edges that have been executed. So, even if a block is contained in the compiled code, all unexecuted edges to that block must fall back to the interpreter so that the profiling data is updated. Our tracebased JIT compiler does that as shown in Figure 5 (e) where block 7 is contained in the trace graph but the unexecuted edge from block 4 to block 7 deoptimizes to the trace recording interpreter. 


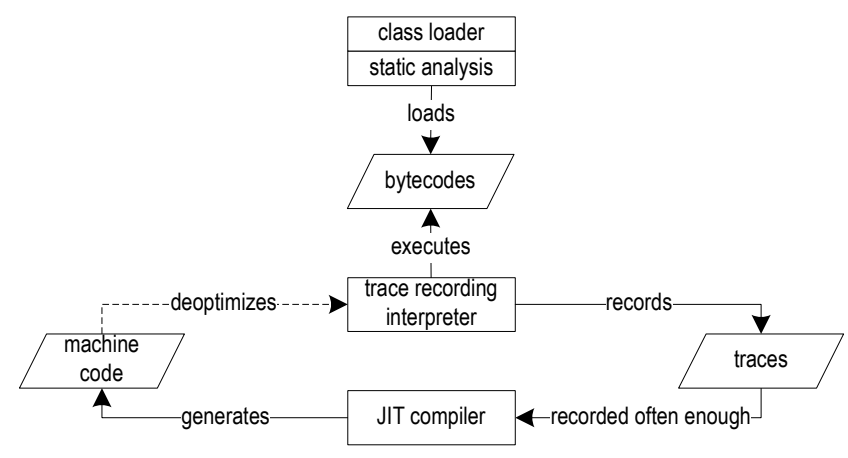

Figure 6. Runtime system for recording coverage information

- If code needs to be executed that has not been executed before, the system must fall back to the instrumented interpreter or to the baseline compiled code so that the corresponding profiling data is updated.

Thus, our approach could, for example, also be used for Oracle's method-based HotSpot server compiler:

- The HotSpot interpreter is already instrumented to record profiling data for the server compiler. However, to maximize the startup performance, profiling data is only recorded after a method was executed a certain number of times. This threshold would have to be set to zero so that the profiling data is recorded from the beginning.

- In most cases, the server compiler already avoids compiling unexecuted code and unexecuted control flow edges. However, in a few cases such as when on-stack-replacement (OSR) [20] is used to optimize a long running loop, unexecuted code may get compiled. This would have to be changed so that only executed Java bytecode is compiled to machine code.

- If code has to be executed that was not compiled yet, the machine code generated by the server compiler already deoptimizes to the interpreter and updates the profiling data accordingly. So, there would not be any changes necessary in this area.

Our requirements for determining code coverage are few so that it should be easy to fulfill them in most modern VMs that use an instrumented interpreter or baseline compiler to record profiling information. However, our approach is not compatible with samplebased profiling.

\subsection{Computing Code Coverage}

There are many different code coverage metrics such as method coverage, basic block coverage, instruction coverage, edge coverage, or path coverage [16]. Our paper concentrates on deriving instruction and edge coverage from the recorded traces. In most cases, we could also derive path coverage information from the recorded trace information. However, if partial traces were recorded, the path before the partial traces might be indeterminable because partial traces do not start at a trace anchor. If multiple paths from the enclosing trace anchor to the start of the partial trace are possible, we cannot decide which path should be considered as the predecessor of the partial trace.

Similar to the profiling data for a method-based JIT compiler, the recorded traces are stored in main memory while the VM is running. When the VM exits (or upon user request) we compute the code coverage information and write it to a file. Figure 7 shows how we compute the code coverage information from the recorded traces. At first, we do some pre-processing where we analyze the method bytecodes to compute all control flow edges.

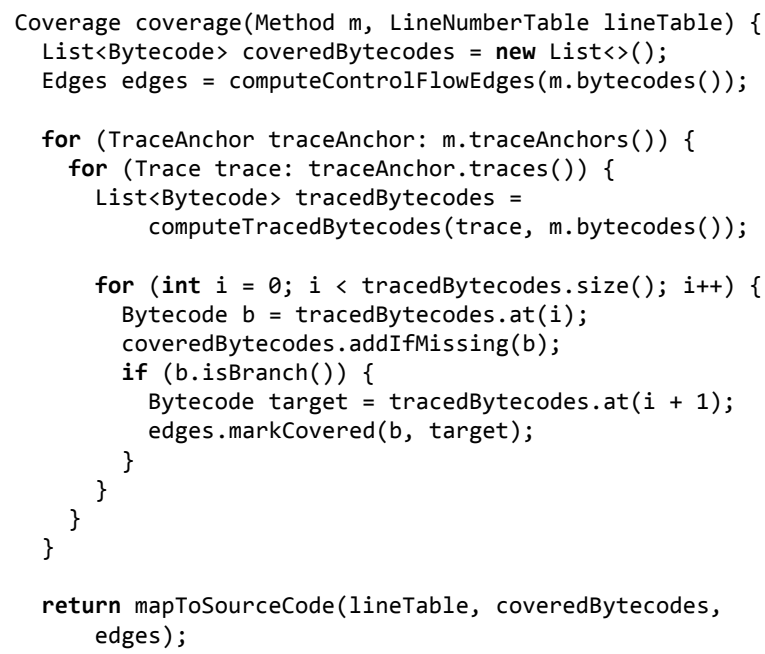

Figure 7. Computing code coverage from recorded traces
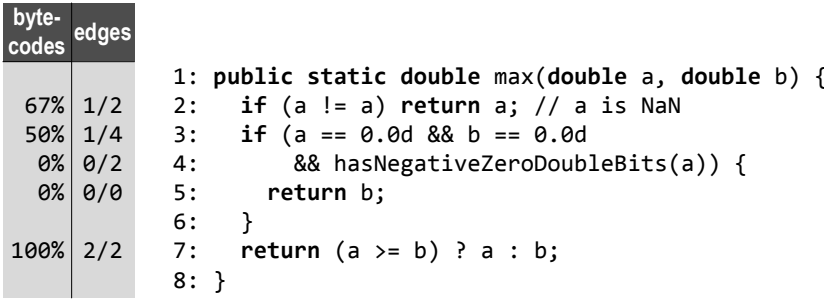

(a) coverage for the initial trace graph
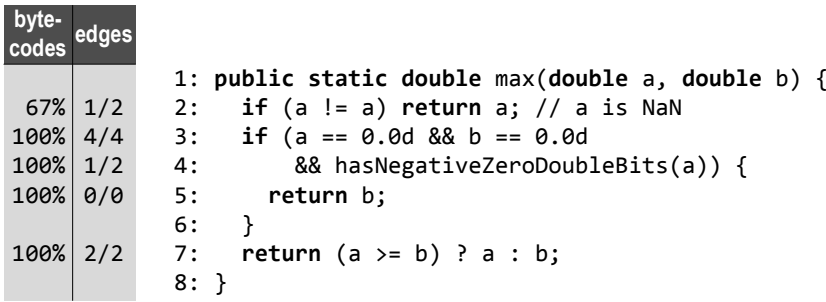

Figure 8. Line coverage for the method Math. $\max ()$

Then, we query all trace anchors for the current method and iterate over all their traces. The recorded traces only contain the raw information about the recorded control flow decisions, see Figure 4 , so that we explicitly simulate the control flow decisions of each trace to determine which bytecodes it covers. Every method bytecode that was covered by a trace is then marked as covered. Furthermore, we check for every covered bytecode if it is a branching instruction and if so, we mark the control flow edge that remains inside the trace as covered.

After processing all traces, the covered bytecodes and control flow edges are mapped to the source code using the line number table within the class file. The resulting coverage data is stored to a file so that the actual visualization of the covered code parts can be performed offline. Figure 8 shows exemplary the line and edge coverage for the trace graphs in Figure 5 (c) and (e). Some of the lines are only partially covered as not all bytecodes/edges have been executed. For a more in-depth look at the recorded code coverage, it is also possible to visualize the data on the bytecode-level. 


\begin{tabular}{|c|c|c|c|c|}
\hline & $\begin{array}{l}\text { source code } \\
\text { instrumentation }\end{array}$ & $\begin{array}{l}\text { offline bytecode } \\
\text { instrumentation }\end{array}$ & $\begin{array}{l}\text { online bytecode } \\
\text { instrumentation }\end{array}$ & our approach \\
\hline works without access to source code & no & yes & yes & yes \\
\hline works with classes created at run-time & no & no & yes & yes \\
\hline does not affect reflection & depends on the implementation & depends on the implementation & depends on the implementation & yes \\
\hline negative impact on peak performance & high & high & high & low \\
\hline works for very large methods & no & no & no & yes \\
\hline possible to instrument all JDK classes & no & no & no & yes \\
\hline can instrument container files (.jar, .war) & $\begin{array}{l}\text { only if instrumented before } \\
\text { packaging }\end{array}$ & yes & yes & yes \\
\hline can instrument signed container files & $\begin{array}{l}\text { only if instrumented before } \\
\text { packaging }\end{array}$ & $\begin{array}{l}\text { only if instrumented before } \\
\text { packaging }\end{array}$ & yes & yes \\
\hline supports explicitly thrown exceptions & yes & yes & yes & yes \\
\hline supports implicitly thrown exceptions & $\begin{array}{l}\text { only if probes are inserted after } \\
\text { instructions that may throw }\end{array}$ & $\begin{array}{l}\text { only if probes are inserted after } \\
\text { instructions that may throw }\end{array}$ & yes & yes \\
\hline works without additional instrumentation & no & no & no & yes \\
\hline independent of source language & no & yes & yes & yes \\
\hline difficulty to map coverage to source code & low & high & high & high \\
\hline VM independent & yes & yes & yes & no \\
\hline
\end{tabular}

Figure 9. Comparison of code coverage instrumentation techniques

For mapping bytecodes to line numbers, our approach relies on the line number table within the class files. However, this table is only present when the class files are generated with debugging information. If no line number table is available, the coverage information can still be visualized on the bytecode-level.

The recorded traces also contain further data that could be visualized, such as execution counts, type information, and information about the call targets of virtual calls. However, unlike the code coverage information, this data is not accurate and only represents the data observed during the executions in the trace recording interpreter.

\subsection{Comparison to Other Code Coverage Techniques}

Figure 9 shows different instrumentation techniques that can be used to record code coverage information for Java.

When source code is instrumented, probes are inserted at the source code level and the modified source code is compiled to Java bytecode. Instrumenting on this level greatly simplifies the mapping of the recorded code coverage information to the source code so that this approach can even show within lines which source code parts have been executed. All other techniques must use the line number table within the Java class files to map the executed bytecodes to source code lines. The biggest disadvantage of source code instrumentation is the language dependency, especially as there are several languages that can be compiled to Java bytecode. So, a separate implementation is necessary for every language.

Bytecode instrumentation inserts probes into the bytecodes after the source code has been compiled to Java bytecode. This is either done in an offline preprocessing step or during execution of the Java program. Offline bytecode instrumentation directly modifies the class files, which reduces the run-time overhead. However, it causes problems for signed class file containers as the signature no longer matches the modified class files. Online bytecode instrumentation attaches an agent to the JVM Tool Interface [27] and modifies the bytecodes after they have been loaded by the VM class loader. This makes code coverage tools convenient to use as it allows transparent instrumentation of class file containers such as .jar or .war files. Furthermore, the attached agent can be notified when the application throws an implicit exception. This simplifies recording exact code coverage information in case of implicit exceptions. Bytecode instrumentation must use the line number table within the Java class files to map the executed bytecodes to source code lines. However, several bytecodes may map to the same line so that it can be hard to determine which parts of a line have been executed. So, it is common to report a coverage percentage for those lines where not all parts have been executed $[1,2,6]$.

All approaches that explicitly add instrumentation to the code suffer from the problem that the JIT compiler generates machine code for the inserted probes. So, the probes are also executed when the code is already compiled, which decreases the peak performance. Compiling the probes also increases both the JIT compilation time and the size of the generated machine code. Our approach avoids this issue as it does not need any additional instrumentation. Instead, we reuse the profiling data that is recorded for JIT compilation by already existing instrumentation. This allows retaining almost full peak performance so that code coverage can even be recorded when the application is already deployed on a production system.

Another advantage of our approach is that we also record exact code coverage in case of implicit exceptions. For performance reasons, other approaches often only insert probes at the end of every basic block so that the whole basic block is marked as executed when this probe is executed. When an implicit exception happens before the probe, then the whole block is not marked as covered although some instructions were executed.

Some implementations of source code or bytecode instrumentation add methods and fields to the instrumented classes so that the bytecodes can update the coverage data more efficiently. This reduces the instrumentation overhead but can have undesired effects as those fields are visible using reflection. Modifying top-level classes such as java.lang. Object may also cause serious problems because the VM may rely on a specific object layout for those classes. So, instrumenting an application and all its libraries in such a way is not always possible. Our approach is transparent and does not modify the executed application in any way.

The only drawback of using profiling data to compute code coverage information is that the technique is VM-dependent as every VM records profiling data in a different way. 


\subsection{Code Coverage Tools}

For Java, there are many open source and commercial code coverage tools of which we list only a few popular ones.

Source code instrumentation is, for example, used by CodeCover [3] (open source) and Clover [4] (commercial). Because of instrumenting the source code these tools are language dependent but have the advantage that they can map the executed probes to the source code more easily.

EMMA [1] and Cobertura [2] are both open source tools that use bytecode instrumentation and both have been popular for several years. However, EMMA and Cobertura are no longer under active development and therefore they do not support the new language features that were introduced with Java 7. $\mathrm{JaCoCo}[6]$ is a still supported open source tool that does both online and offline bytecode instrumentation and which supports the latest Java features. The online bytecode instrumentation greatly simplifies the use of this tool as it is sufficient to add just one command line flag to instrument a Java application transparently. EclEmma [5] is an open source Eclipse plugin that can directly visualize the coverage information in Eclipse. Previously, EclEmma used EMMA to record the code coverage information but then switched to JaCoCo because EMMA was no longer under active development and did not support the latest Java features.

Many code coverage tools do not support implicitly thrown exceptions so that the coverage information is inaccurate when such an exception is thrown. For example, EMMA and JaCoCo assume that all instructions before a probe are executed as one atomic unit so that those instructions are only marked as covered after the successive probe has been executed. This reduces the instrumentation overhead and increases the performance but has the drawback that the code coverage information is inaccurate in the case of implicitly thrown exceptions.

Figure 10 (a) shows the source code and Figure 10 (b) the bytecode of a simple method that does some synchronization and returns the absolute value of a static field. When the code is instrumented with JaCoCo, the bytecode in black type shown in Figure 10 (c) is generated. The bytecodes 0 to 3 are used to get an array of boolean values that contains the recorded coverage data for the current method. This array is then stored in a local variable that is accessed by the probes that update the code coverage information (see bytecodes 7 to 10,19 to 22,29 to 32 , and 37 to 40 ).

In this specific example, the bytecode instrumentation causes an unexpected performance bug. Figure 10 (b) shows that no instruction in the synchronized block could throw an exception (bytecodes 3 to 14). JaCoCo inserts three probes in the synchronized block and all execute an array store to update the coverage array. However, the array store instruction bastore may throw an exception. Although those bytecodes are valid according to the JVM specification [26], this results in a construct that a Java source to class file compiler such as javac would not generate. Those compilers always add a catch-all exception handler that unlocks the monitor in case of an exception and rethrows the exception, see bytecodes 42 to 45 in gray type in Figure 10 (c).

A Java JIT compiler can always decide to avoid compiling certain bytecode constructs for performance or implementation reasons. So, before compiling a method or method parts, the HotSpot compilers validate that the bytecode fulfills certain invariants and assumptions, especially when monitors are used. In this example, it turns out that the bytecodes 10, 22, and 32 may throw an ArrayIndexOutOfBoundsException that would unwind the method although there are still locked monitors remaining. HotSpot does not allow this behavior for compiled code so that the bytecodes are not compiled by the JIT compiler and are executed in the interpreter instead. The interpreter is prepared for such byte-

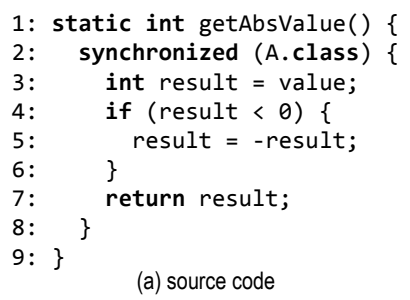

(a) source code

$$
\begin{aligned}
& 0 \text { ldc A.class } \\
& 2 \text { monitorenter } \\
& 3 \text { getstatic value } \\
& 6 \text { istore result } \\
& 7 \text { iload result } \\
& 8 \text { ifge } 14 \\
& 11 \text { iload result } \\
& 12 \text { ineg } \\
& 13 \text { istore result } \\
& 14 \text { iload result } \\
& 15 \text { ldc A.class } \\
& 17 \text { monitorexit } \\
& 18 \text { ireturn } \\
& \text { (b) uninstrumented bytecode }
\end{aligned}
$$

0 invokestatic \$jacocoInit

3 astore coverageData

4 ldc A.class

6 monitorenter

7 aload coverageData

8 iconst 1

9 iconst 1

10 bastore

11 getstatic value

14 istore result

15 iload result

16 iflt 26

19 aload coverageData

20 iconst 2

21 iconst 1

22 bastore

23 goto 33

26 iload result

27 ineg

28 istore result

29 aload coverageData

30 iconst 3

31 iconst 1

32 bastore

33 iload result

34 ldc A.class

36 monitorexit

37 aload coverageData

38 iconst 4

39 iconst 1

40 bastore

41 ireturn

// catch all exceptions

42 ldc A.class

44 monitorexit

45 athrow

(c) instrumented bytecode codes and in case of an exception it unlocks all remaining monitors before unwinding the stack frame.

The trace-based compiler that is used in this paper also checks the same invariants and assumptions as the method-based HotSpot compilers. So, none of the HotSpot compilers would generate machine code for this instrumented method so that this method is only executed in the interpreter. Similar cases happened for a few methods in the evaluated benchmark suites (see Section 4) so that this affected the performance of some benchmarks when JaCoCo is used to determine code coverage. We also checked this for the code coverage frameworks EMMA and Cobertura, which also use bytecode instrumentation, and they suffer from the same issue.

\subsection{Tuning Startup Performance}

The unmodified trace-based runtime system uses two different interpreters: a normal and a trace recording interpreter. The normal interpreter does not do any tracing and is used during startup for executing the bytecodes. To obtain exact code coverage information, our modified runtime system must only use the trace recording interpreter as shown in Figure 6. This decreases the startup performance because tracing happens more frequently than before.

However, the recorded trace information is more accurate because more traces are being recorded before compiling them to machine code. The trace-based JIT compiler uses the recorded traces for optimistic optimizations so that more accurate information results in fewer invalidated optimizations. So, deoptimization is required less frequently which increases the startup performance.

Therefore, the startup performance depends on how often traces are recorded for a trace anchor before a JIT compilation is triggered as the positive effect of the more accurate trace information 
competes against the negative effect of the higher trace recording overhead. The unmodified trace-based runtime system uses the following thresholds to trigger trace recording and trace compilation:

- The method tracing threshold determines how often a method has to be executed before the execution switches to the trace recording interpreter so that traces starting from the method entry are recorded.

- The method traces compilation threshold determines how often traces are recorded for a trace anchor at a method entry before those traces are merged and compiled to optimized machine code.

- The loop tracing threshold determines how often a loop header has to be executed before the execution switches to the trace recording interpreter so that traces starting from the loop header are recorded.

- The loop traces compilation threshold determines how often traces are recorded for a trace anchor at a loop header before those traces are merged and compiled to optimized machine code.

Figure 11 shows the thresholds that are used in the unmodified and the modified runtime systems. The fairly high thresholds are used to avoid that infrequently executed method and loop traces are compiled to machine code. For example, the unmodified runtime system compiles loops before their surrounding method if the loop body is executed at least 6.5 times per method invocation. At the first glance, those values may seem high but they are already lower than the thresholds that are used in the method-based HotSpot server compiler. Furthermore, we use a complexity metric that estimates how many different traces can be recorded for a specific trace anchor [17]. Depending on the number of possible traces, the compilation thresholds are reduced so that compilation is triggered earlier.

For the code coverage configurations, the tracing thresholds for method entries and loop headers were reduced to zero, so that only the trace recording interpreter is used. In our configuration tracebased coverage untuned, we mimicked the compilation behavior of the unmodified trace-based compiler by increasing the compilation thresholds accordingly. Depending on the executed application, this can have a significant impact on startup performance because especially loops are trace recorded many times. In our configuration trace-based coverage tuned, we significantly decreased the compilation threshold for loops and we slightly decreased the compilation threshold for method traces. For the benchmarks in the next section, this improves the startup performance to the level of the unmodified trace-based runtime system.

\section{Evaluation}

Our trace-based runtime system was implemented for the IA-32 architecture of Oracle's Java HotSpot VM using the early access version b12 of the upcoming JDK 8 [28]. For benchmarks we used an Intel Core-i5 processor with 4 cores running at $2.66 \mathrm{GHz}$, $4 * 256 \mathrm{~kb}$ L2 cache, $8 \mathrm{MB}$ shared L3 cache, $8 \mathrm{~GB}$ main memory, and with Windows 8 Professional as the operating system. We evaluated our trace-based compiler on the benchmark suites SPECjvm2008 [30] and DaCapo 9.12 Bach [10] and present results for the following configurations:

- The baseline is our unmodified trace-based JIT compiler and all results are normalized to the results of this configuration. In the figures, this configuration is only shown implicitly as the $100 \%$ mark.

\begin{tabular}{|l|c|c|c|}
\cline { 2 - 4 } \multicolumn{1}{c|}{} & $\begin{array}{c}\text { original } \\
\text { trace-based } \\
\text { compiler }\end{array}$ & $\begin{array}{c}\text { trace-based } \\
\text { coverage } \\
\text { untuned }\end{array}$ & $\begin{array}{c}\text { trace-based } \\
\text { coverage } \\
\text { tuned }\end{array}$ \\
\hline method tracing threshold & 3000 & 0 & 0 \\
\hline method traces compilation threshold & 1000 & 4000 & 3000 \\
\hline loop tracing threshold & 25000 & 0 & 0 \\
\hline loop traces compilation threshold & 1000 & 26000 & 4000 \\
\hline
\end{tabular}

Figure 11. Thresholds used for trace recording and JIT compilation

\begin{tabular}{|l|c|c|}
\cline { 2 - 3 } \multicolumn{1}{c|}{} & JaCoCo & our approach \\
\hline instrumentation technique & $\begin{array}{c}\text { online or offline bytecode } \\
\text { instrumentation }\end{array}$ & $\begin{array}{c}\text { no additional } \\
\text { instrumentation }\end{array}$ \\
\hline line coverage & yes & yes \\
\hline detects partially covered lines & yes & yes \\
\hline edge coverage & yes & yes \\
\hline path coverage & yes & partially ${ }^{2}$ \\
\hline supports explicit exceptions & no & yes \\
\hline supports implicit exceptions & no & yes \\
\hline records execution counts & partially ${ }^{3}$ \\
\hline can instrument all classes & $\begin{array}{c}\text { excludes classes on the } \\
\text { boot classpath }\end{array}$ & yes \\
\hline
\end{tabular}

${ }^{1}$ we use online instrumentation as it allows us to instrument the application transparently

${ }^{2}$ some information is lost for partial traces

${ }^{3}$ only recorded while code is executed in the interpreter

Figure 12. Features of the evaluated tools

- The configuration JaCoCo shows the performance of the JaCoCo [6] code coverage tool in the version 0.62 when used with our unmodified trace-based runtime system. We use JaCoCo's online bytecode instrumentation mechanism as this greatly simplifies instrumenting the benchmark suites which do a significant amount of class loading at run-time.

- The configuration trace-based coverage untuned uses the tracebased compiler and our modified runtime system to record code coverage information. So, the trace recording interpreter records traces from the beginning. Eventually, the recorded traces are compiled to optimized machine code. The threshold for compiling the traces is as shown in Figure 11.

- The configuration trace-based coverage tuned also uses the trace-based compiler and our modified runtime system to record code coverage information. However, we reduced the compilation thresholds as described in Figure 11 so that the startup performance improves because traces are compiled earlier.

- The configuration HotSpot server shows the unmodified Java HotSpot server compiler to compare the startup and peak performance of our trace-based compiler with a highly-optimized method-based JIT compiler. The HotSpot server compiler is explicitly designed for peak performance and performs significantly more optimizations than our trace-based compiler, e.g., escape analysis and sophisticated loop optimizations.

Figure 12 shows a comparison between the features of $\mathrm{JaCoCo}$ and our approach. One important difference between $\mathrm{JaCoCo}$ and our approach is that JaCoCo does not record code coverage information for the classes on the boot classpath, while our approach records code coverage information for all loaded classes. So, JaCoCo also excludes all classes defined in the rt.jar file such as String and ArrayList which are used frequently. Code coverage information for those classes is usually not particular interesting but it can for example be used to identify the JDK parts that are used 


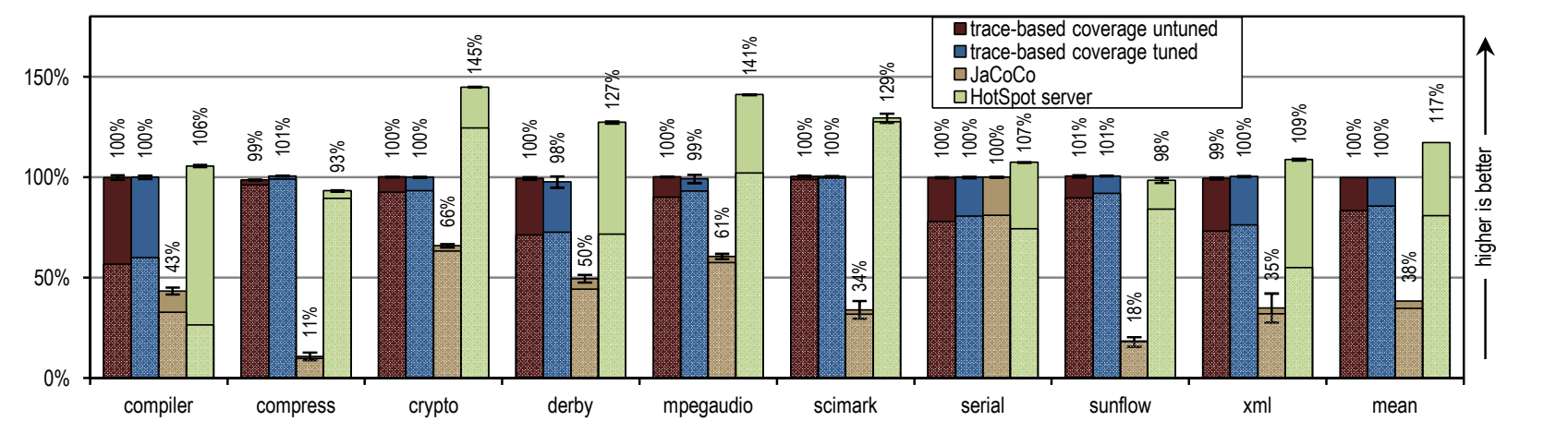

Figure 13. SPECjvm2008: startup and peak performance

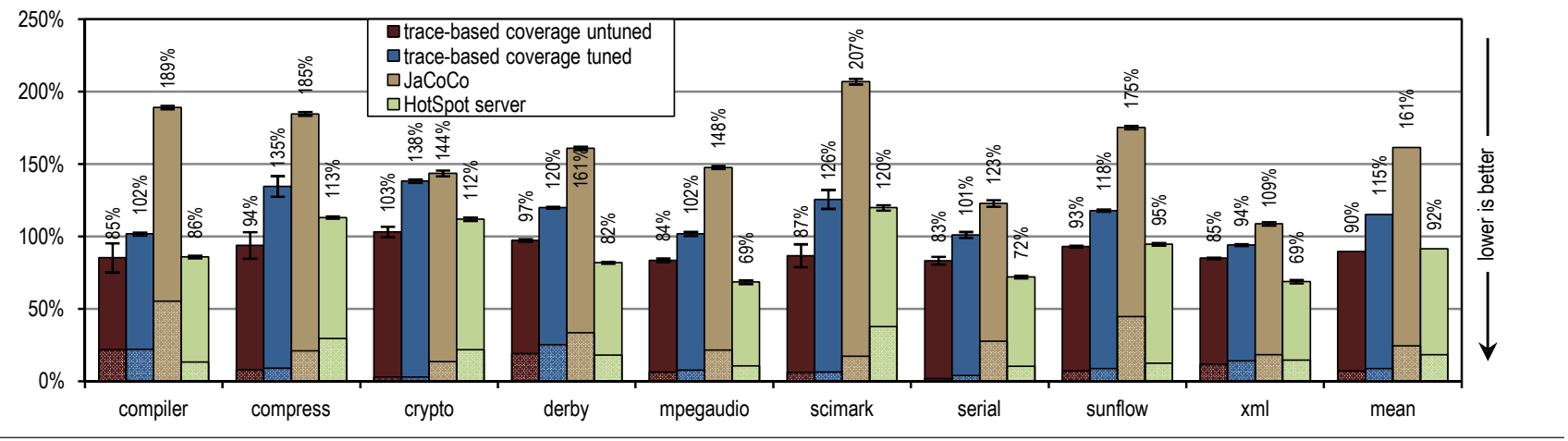

Figure 14. SPECjvm2008: amount of generated machine code

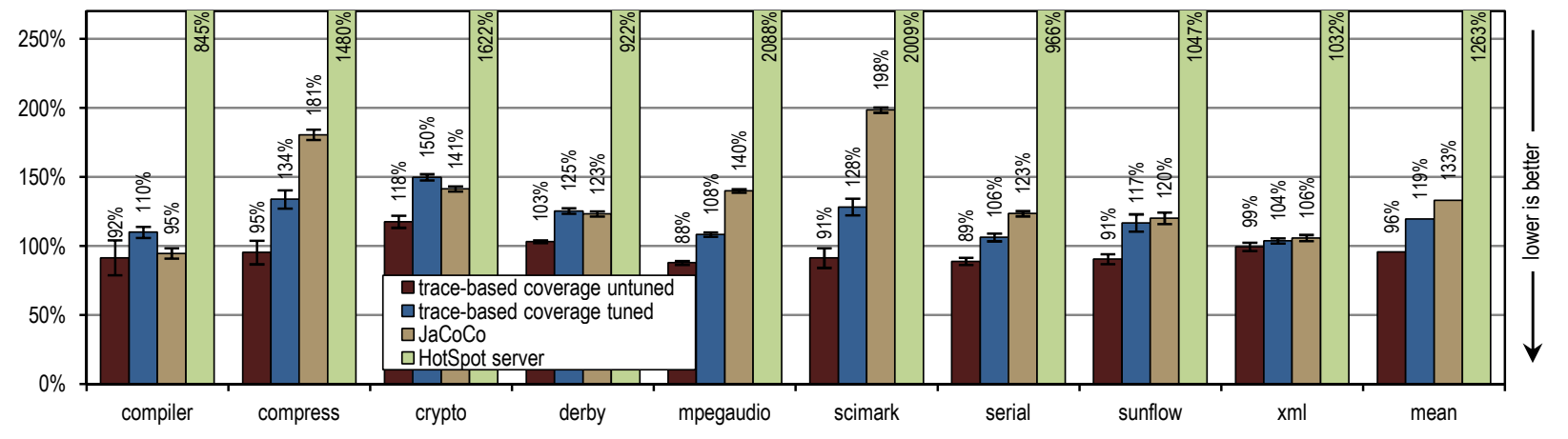

Figure 15. SPECjvm2008: time required for JIT compilation

by an application. Besides that, we tried to stress the code coverage instrumentation as much as possible so that we did not exclude any application classes from the collection of code coverage information. So, for many applications it would be possible to further reduce the amount of instrumentation by limiting code coverage to a minimum set of interesting classes.

Each benchmark suite in the following sections was executed 15 times and we report the average of those results along with the $95 \%$ confidence interval.

\subsection{SPECjvm2008}

The SPECjvm2008 benchmark suite contains nine benchmark categories that measure peak performance. Furthermore, we measure startup performance by executing each benchmark for one benchmark operation in a new VM. Next to the individual results for each benchmark category, we show the geometric mean of all benchmark categories. A heap size of $1024 \mathrm{MB}$ was used for all measurements.
Figure 13 shows the performance results for the SPECjvm2008 benchmark suite. In this diagram, the startup and the peak performance for each benchmark category are shown on top of each other. Both runs are shown relative to the fastest run of the baseline.

In comparison to the unmodified trace-based compiler, both trace-based code coverage configurations succeed in preserving full peak performance. This is the case because the compiled machine code does not contain any instrumentation so that there is no overhead when executing the compiled code. In terms of startup performance, our configuration trace-based coverage tuned reaches the same performance level as the unmodified trace-based JIT compiler (not shown in the diagram). Tuning the compilation thresholds reduces the impact of trace recording on startup performance so that the startup performance of all benchmarks improves in comparison to the configuration trace-based coverage untuned.

When the bytecodes of the benchmarks are instrumented using $\mathrm{JaCoCo}$ then the added probes have a significantly negative effect on the peak performance because they are compiled to ma- 


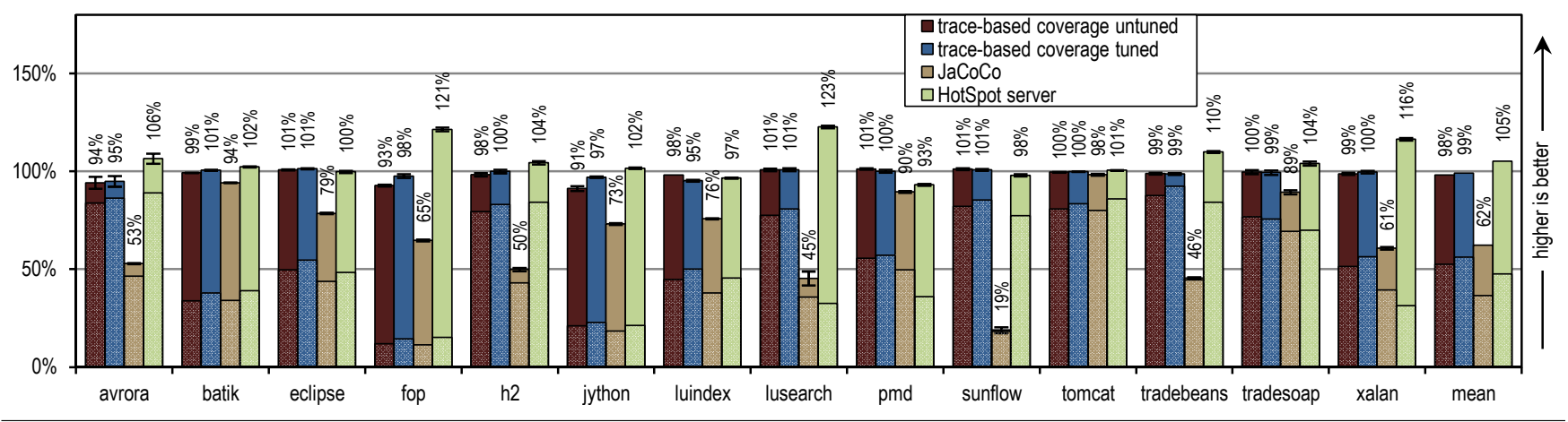

Figure 16. DaCapo 9.12 Bach: startup and peak performance

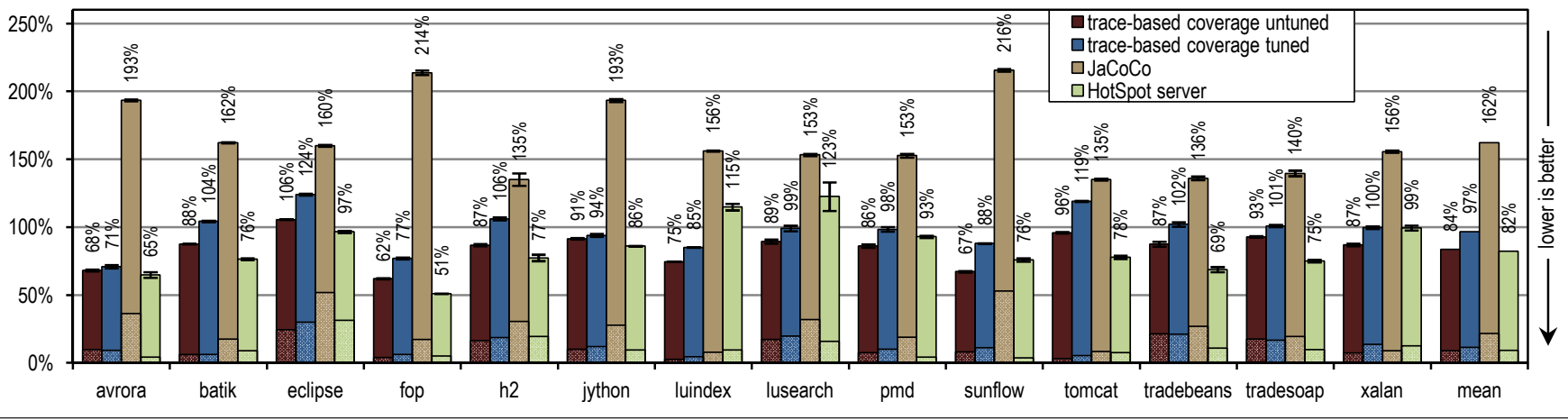

Figure 17. DaCapo 9.12 Bach: amount of generated machine code

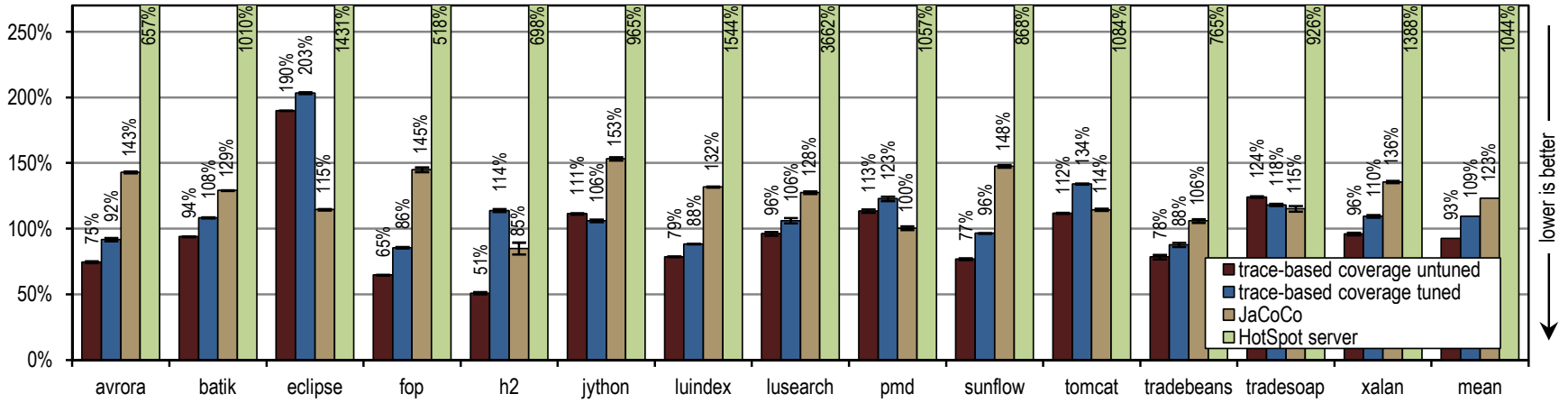

Figure 18. DaCapo 9.12 Bach: time required for JIT compilation

chine code and executed also in compiled code. One exception is the benchmark serial, which mainly executes JDK methods in its performance-critical code parts. JDK methods are not instrumented by $\mathrm{JaCoCo}$ and the probes within the actual benchmarking logic do not impact the peak performance of this benchmark.

The dark bars in Figure 14 show the total amount of generated machine code, while the lighter/textured bars indicate the amount of machine code that was invalidated because optimistic optimizations deoptimized too frequently. The configuration trace-based coverage untuned generates less machine code than the unmodified trace-based compiler because it does record traces for a longer time before compiling them. So, the recorded trace information is more accurate and it is less likely that the compiled machine code deoptimizes and has to be invalidated and recompiled because of too frequent deoptimization. In contrast to that, the configuration trace-based coverage tuned generates more machine code than the unmodified trace-based compiler because of two factors. Traces are recorded fewer times so that the trace information is less accurate and deoptimization and invalidation of machine code occurs more frequently. Second, the SPECjvm2008 benchmarks are loop intensive and due to the lower compilation threshold for loops, more loops are compiled during startup. Some of this generated code becomes redundant later when the enclosing method traces are compiled, and the loop traces may get inlined.

When the benchmarks are instrumented with $\mathrm{JaCoCo}$, the amount of generated machine code increases heavily because the instrumentation code is also compiled.

A similar behavior can also be seen in Figure 15, which shows the amount of time required for JIT compilation. However, the increase in compilation time is not as significant because the instrumentation code is simple and hardly complicates the compilation.

\subsection{DaCapo 9.12 Bach}

The DaCapo 9.12 Bach benchmark suite consists of 14 objectoriented applications. We executed each benchmark for 20 times with the default data size so that the execution time converges 
and we show the first and the fastest run for each benchmark. The first run shows the startup performance of the VM, while the fastest run shows the peak performance. Furthermore, we present the geometric mean of all results. A heap size of $1024 \mathrm{MB}$ was used for all measurements.

Figure 16 shows the performance results for the DaCapo 9.12 Bach benchmark suite. In this diagram, the startup and the peak performance for each benchmark category are shown on top of each other. Both runs are shown relative to the fastest run of the baseline.

On average, both code coverage configurations that use our trace-based runtime system achieve the same peak performance as the baseline. However, for a few benchmarks such as avrora, jython, and luindex our approach for recording code coverage information impacts peak performance. This happens because different parts of the execution are trace recorded due to the changed thresholds. So, the recorded trace information also covers code that is only required during startup. Those differences in the trace information may result in less effective compiler optimizations. However, the JaCoCo instrumented run is still far slower despite not recording any coverage information for classes on the boot classpath. The main exception is the benchmark tomcat which spends most of its time in native functions where neither $\mathrm{JaCoCo}$ nor our approach places any instrumentation.

In terms of startup performance, the configuration trace-based coverage tuned reaches the same level as the unmodified tracebased JIT compiler (not shown in the diagram). The benchmark tradesoap is the only one that does not profit from tuning startup performance because the reduced compilation threshold results in more frequent deoptimization.

The dark bars in Figure 17 show the total amount of generated machine code, while the lighter/textured bars indicate the amount of machine code that was invalidated because optimistic optimizations deoptimized too frequently. The configuration trace-based coverage untuned generates less machine code than the unmodified trace-based compiler because the recorded trace information is more accurate so that less code has to be invalidated and recompiled. By decreasing the threshold for JIT compilation, the configuration trace-based coverage tuned increases the startup performance but also generates more machine code. However, due to the more accurate trace information (traces are still recorded 3 to 4 times more often before compilation), deoptimization and recompilation of traces is required less frequently than in the unmodified trace-based JIT compiler. The configuration $\mathrm{JaCoCo}$ generates by far the largest amounts of machine code because the instrumentation code is also compiled.

Figure 15 shows the time required for JIT compilation. For most benchmarks, the instrumentation added by JaCoCo increases the time required for JIT compilation. Exceptions are the benchmarks $h 2$ and tradesoap where several trace anchors cannot be compiled because the instrumentation violates invariants assumed by HotSpot, see Section 3.4. Especially for $h 2$, this also impacts the startup and peak performance.

Another interesting case can be observed for the benchmark eclipse. Both trace-based coverage configurations require significantly more time for JIT compilation than the unmodified tracebased runtime system. This happens because the different trace information leads to different trace inlining decisions. Therefore, some compilation units get significantly larger, which increases the compilation time by a non-linear factor. However, it also results in slightly better optimized code so that the peak performance increases.

\subsection{Further Evaluations}

Figure 19 shows some statistics about the recorded traces when the benchmarks suites are executed with the trace-based baseline and

\begin{tabular}{|l|l|r|r|}
\cline { 3 - 4 } \multicolumn{2}{c|}{} & baseline & $\begin{array}{c}\text { trace-based } \\
\text { coverage tuned }\end{array}$ \\
\hline \multirow{2}{*}{ total trace size in MB } & SPECjvm2008 & 0,30 & 2,68 \\
\cline { 2 - 4 } number of full traces & DaCapo 9.12 Bach & 1,17 & 4,51 \\
\hline \multirow{2}{*}{ number of partial traces } & SPECjvm2008 & 4052 & 31953 \\
\cline { 2 - 4 } & DaCapo 9.12 Bach & 12651 & 45351 \\
\hline & SPECjvm2008 & 449 & 242 \\
\cline { 2 - 4 } & DaCapo 9.12 Bach & 861 & 423 \\
\hline
\end{tabular}

Figure 19. Trace recording statistics

the configuration trace-based coverage tuned. When code coverage information is being recorded, a significantly higher number of full traces (i.e. those that start at trace anchors) is being recorded. This also increases the amount of memory that is used by the traces but on average the traces still use only a few megabytes of memory. So, it should be possible to record code coverage for large server applications without a significant increase in memory usage.

Figure 20 shows for the individual benchmarks how many trace anchors could not be compiled because the instrumentation added by JaCoCo violates some HotSpot assumption. For the SPECjvm2008 benchmark suite only some trace anchors in the benchmark derby are not jitted because of those HotSpot assumptions. The DaCapo 9.12 Bach benchmark suite is significantly more complex than the SPECjvm2008 benchmark suite [17], so that several benchmarks are affected.

\subsection{Discussion of Results}

In comparison to existing code coverage frameworks such as JaCoCo, our approach does not add any instrumentation to the application. This avoids that the machine code generated by the JIT compiler contains any instrumentation that would degrade performance and unnecessarily increase the amount of generated machine code. Due to the changed trace recording and compilation thresholds, our trace-based configurations record different trace information. This may change the optimizations used by the JIT compiler and can affect performance. However, our approach still has a very low performance and memory overhead so that it can also be used to record code coverage in daily operation.

We also validated our JaCoCo performance results, by executing the JaCoCo-instrumented benchmarks with an unmodified version of the HotSpot server compiler. On average, the results were similar to those seen with our trace-based JIT compiler.

\section{Related Work}

The approaches related to our work can be roughly divided into two categories. The first category tries to place instrumentation more selectively so that fewer probes are required. The second category removes instrumentation as soon as it is no longer needed.

\subsection{Selective Instrumentation}

Ball et al. [9] propose an efficient algorithm for path profiling. They add instrumentation at certain positions in a method so that the instrumentation computes a path identifier when executed. This path identifier exactly identifies the executed path. Our trace recording interpreter records control flow decisions and other information such as observed types while the application is being executed. This additional information is valuable for aggressive and optimistic compiler optimizations and helps us to increase the peak performance.

Agrawal [7, 8] does a variant of dominator computation to identify leaf blocks that must be instrumented to record code coverage information. Instrumenting only leaf blocks greatly reduces the 


\begin{tabular}{|l|c|c|c|c|c|c|c|c|c|}
\hline SPECjvm2008 & compiler & compress & crypto & derby & mpegaudio & scimark & serial & sunflow & xml \\
\hline not compiled trace anchors & 0 & 0 & 0 & 39 & 0 & 0 & 0 & 0 & 0 \\
\hline compiled trace anchors & 2508 & 384 & 566 & 1627 & 530 & 350 & 736 & 619 & 1451 \\
\hline
\end{tabular}

\begin{tabular}{|c|c|c|c|c|c|c|c|c|c|c|c|c|c|c|}
\hline DaCapo 9.12 Bach & avrora & batik & eclipse & fop & h2 & jython & luindex & lusearch & pmd & sunflow & tomcat & tradebeans & tradesoap & xalan \\
\hline not compiled trace anchors & 5 & 0 & 50 & 2 & 25 & 0 & 5 & 3 & 0 & 0 & 25 & 32 & 63 & 6 \\
\hline compiled trace anchors & 664 & 1573 & 5377 & 1325 & 1059 & 1648 & 618 & 571 & 1835 & 454 & 3227 & 2018 & 3839 & 1345 \\
\hline
\end{tabular}

Figure 20. Not compiled trace anchors because of the bytecode instrumentation added by JaCoCo

amount of instrumentation. However, in case of exceptional control flow, this technique is less efficient because it requires every potentially exception throwing instruction to be instrumented. Otherwise, an exception could leave the current block or method without marking already executed code as covered. Our approach is complementary as we do not add any instrumentation to record code coverage. Instead, we use the already existing tracing infrastructure in the interpreter that records profiling data, in our case traces. This avoids any instrumentation in the compiled code and thus minimizes the impact on peak performance.

\subsection{Disposable Instrumentation}

Another approach that is related to ours is to reduce the instrumentation overhead by removing the instrumentation as soon as possible. Pavlopoulou et al. [29] instrument the bytecodes of Java applications to record coverage information. Then, the instrumented application is executed multiple times with different parameters. Whenever the application finishes running, all executed and therefore no longer needed probes are removed from its class files. This reduces the amount of instrumentation with every execution.

Tikir et al. [31] combine selective instrumentation and removal of no longer needed instrumentation to decrease the overhead of determining code coverage. They dynamically instrument binary code while it is executed to record code coverage information and - similar to Agrawal - they use use dominator tree information to reduce the number of inserted probes. Their system periodically checks if there are any probes that are no longer needed and can be removed. So, the amount of instrumentation reduces gradually without stopping the executed application.

Kumar et al. [23, 24] developed a framework for dynamic instrumentation of binary code. They also adapted their tool for the Jikes RVM so that Java applications can be instrumented on the machine code level. The machine code of the Java application is generated by the Jikes JIT compiler which keeps track of which machine code instructions map to which bytecodes. Using this information, they can map positions within the machine code to bytecode, and also to source code if the class file contains a line number table. Probes can be added and removed at run time and are implemented as fast breakpoints [22] that branch to the instrumentation code.

Chilakamarri et al. [11, 12] use bytecode instrumentation to record code coverage information. They modified the Kaffe JVM interpreter in such a way that the instrumentation bytecodes are overwritten with nop instructions once they have been executed. This effectively removes the instrumentation immediately after execution and increases the performance. However, when the Kaffe JIT compiler is used, they only support method coverage which is simple to implement.

Similarly to Tikir et al, Li et al. [25] combine selective instrumentation and removal of no longer needed instrumentation to decrease the overhead of code coverage instrumentation. They propose to do an offline analysis step with their super nested block algorithm, which aims at reducing the number of required probes and is similar to the dominator-based approach of Agrawal. The results of this offline analysis is then used when instrumenting the application at run time, so that probes do not need to be added to every basic block. After a probe was executed, they remove it in a similar way as Tikir et al.

Dmitriev [13, 14] uses the hotswapping API of the HotSpot JVM to dynamically instrument Java applications. The hotswapping API provides mechanisms to replace methods and classes with different versions at run time. So, this approach can be used to add instrumentation to an application but it can also be used to remove no longer needed instrumentation. Hotswapping has a certain runtime overhead but when focusing on peak performance, the possibility to remove the instrumentation outweighs this overhead.

Forax [15] uses bytecode instrumentation to insert code coverage probes into Java applications. The probes are modeled as Java 7 invokedynamic bytecodes. When an invokedynamic bytecode is executed for the first time, the VM invokes a developer-defined bootstrapping method that resolves the target method. For subsequent executions, the target method is cached so that it can be invoked directly. Forax exploits this behavior and updates the code coverage information in the bootstrapping method and returns a reference to a method that does not perform any operations. This ensures that the code coverage information is updated properly, while the runtime overhead is kept small because subsequent executions invoke an empty method.

Our approach is different from all those approaches as we avoid the explicit instrumentation of applications by reusing the already existing tracing infrastructure of our trace recording interpreter. Because we do not add any instrumentation, we also do not have to take care of removing it later. Instead, hot code parts are compiled by the JIT compiler and the compiled code never contains any instrumentation. Thus, our approach gives us code coverage information while avoiding the instrumentation runtime overhead.

\section{Future Work}

Our approach should be applicable to most of today's highperformance VMs that have an aggressive JIT compiler and an already instrumented interpreter or baseline compiler that records profiling data. We decided to implement our approach for a tracebased JIT compiler instead of for a method-based compiler, because the recorded traces are path-based and contain more information than the profiling data recorded for a method-based compiler. From a more practical point of view, the next logic step would be an implementation of our approach for Oracle's HotSpot server compiler, which is one of the most used Java JIT compilers. The HotSpot VM already contains a suitable interpreter that records the required profiling data but there are a few situations in which the HotSpot server compiler generates machine code for not executed bytecodes. Still, it should be fairly straightforward to apply the changes proposed in Section 3.1.

Another important field is visualizing the coverage information. All commonly used code coverage tools visualize the code coverage in a user friendly and intuitive way. While we prototypically implemented a basic visualization to validate the recorded data, it still lacks most convenience features. 


\section{Conclusions}

We presented a novel runtime system which allows us to obtain code coverage information without explicitly instrumenting an application. Instead, we derive exact code coverage information from the profiling data that is already recorded for an optimizing JIT compiler. This gives us coverage information almost for free while avoiding instrumentation in the compiled code parts and thus keeping the run-time overhead to a minimum.

While we implemented our approach for a variant of the HotSpot VM that uses a trace-based JIT compiler, our approach is general enough to be also applicable to most other modern VMs. Measurements showed that our approach hardly affects the performance of applications so that it can also be used in daily operation systems.

\section{Acknowledgments}

This work has been supported by the Austrian Science Fund (FWF): project number P 22493-N18. Oracle and Java are registered trademarks of Oracle and/or its affiliates. Other names may be trademarks of their respective owners.

\section{References}

[1] EMMA: a free Java code coverage tool, 2005. http://emma. sourceforge.net/.

[2] Cobertura, 2010. http://cobertura.sourceforge.net/.

[3] CodeCover, 2011. http://codecover.org/.

[4] Clover, 2013. Atlassian, Inc. http://www.atlassian.com/ software/clover/.

[5] EclEmma, 2013. http://www.eclemma.org/.

[6] JaCoCo, 2013. http://www.eclemma.org/jacoco/.

[7] H. Agrawal. Dominators, Super Blocks, and Program Coverage. In Proceedings of the ACM SIGPLAN-SIGACT Symposium on Principles of Programming Languages, pages 25-34. ACM Press, 1994.

[8] H. Agrawal. Efficient Coverage Testing Using Global Dominator Graphs. In Proceedings of the ACM SIGPLAN-SIGSOFT Workshop on Program Analysis for Software Tools and Engineering, pages 1120. ACM Press, 1999.

[9] T. Ball and J. R. Larus. Efficient Path Profiling. In Proceedings of the ACM/IEEE International Symposium on Microarchitecture, pages 46-57. IEEE Computer Society, 1996.

[10] S. M. Blackburn, R. Garner, C. Hoffman, A. M. Khan, K. S. McKinley, R. Bentzur, A. Diwan, D. Feinberg, D. Frampton, S. Z. Guyer, M. Hirzel, A. Hosking, M. Jump, H. Lee, J. E. B. Moss, A. Phansalkar, D. Stefanović, T. VanDrunen, D. von Dincklage, and B. Wiedermann. The DaCapo benchmarks: Java benchmarking development and analysis. In Proceedings of the ACM SIGPLAN Conference on ObjectOriented Programming Systems, Languages, and Applications, pages 169-190. ACM Press, 2006.

[11] K.-R. Chilakamarri and S. Elbaum. Reducing Coverage Collection Overhead With Disposable Instrumentation. In Proceedings of the International Symposium on Software Reliability Engineering, pages 233-244. IEEE Computer Society, 2004.

[12] K.-R. Chilakamarri and S. Elbaum. Leveraging Disposable Instrumentation to Reduce Coverage Collection Overhead. Software Testing, Verification \& Reliability, 16(4):267-288, 2006.

[13] M. Dmitriev. Application of the HotSwap Technology to Advanced Profiling. Technical report, Sun Microsystems Laboratories, USA, 2002

[14] M. Dmitriev. Profiling Java Applications Using Code Hotswapping and Dynamic Call Graph Revelation. In Proceedings of the International Workshop on Software and Performance, pages 139-150. ACM Press, 2004.

[15] R. Forax. JSR 292 Goodness: Fast code coverage tool in less than 10k, 2011. https://www . java.net/blog/forax/archive/2011/02/12/ jsr-292-goodness-fast-code-coverage-tool-less-10k.

[16] C. Ghezzi, M. Jazayeri, and D. Mandrioli. Fundamentals of Software Engineering, 2nd Edition. Prentice Hall, 2003.

[17] C. Häubl and H. Mössenböck. Trace-based Compilation for the Java HotSpot Virtual Machine. In Proceedings of the International Conference on the Principles and Practice of Programming in Java, pages 129-138. ACM Press, 2011.

[18] C. Häubl, C. Wimmer, and H. Mössenböck. Evaluation of Trace Inlining Heuristics for Java. In Proceedings of the ACM Symposium on Applied Computing, pages 1871-1876. ACM Press, 2012.

[19] C. Häubl, C. Wimmer, and H. Mössenböck. Context-sensitive Trace Inlining for Java. Computer Languages, Systems and Structures, 2013. To appear.

[20] U. Hölzle and D. Ungar. Optimizing Dynamically-dispatched Calls With Run-time Type Feedback. In Proceedings of the ACM SIGPLAN Conference on Programming Language Design and Implementation, pages 326-336. ACM Press, 1994.

[21] U. Hölzle, C. Chambers, and D. Ungar. Debugging Optimized Code with Dynamic Deoptimization. In Proceedings of the ACM SIGPLAN Conference on Programming Language Design and Implementation, pages 32-43. ACM Press, 1992.

[22] P. B. Kessler. Fast Breakpoints: Design and Implementation. In Proceedings of the ACM SIGPLAN Conference on Programming Language Design and Implementation, pages 78-84. ACM Press, 1990.

[23] N. Kumar, J. Misurda, B. R. Childers, and M. L. Soffa. FIST: A Framework for Instrumentation in Software Dynamic Translators. Technical report, University of Pittsburgh, USA, 2003.

[24] N. Kumar, J. Misurda, B. R. Childers, and M. L. Soffa. Instrumentation in Software Dynamic Translators for Self-Managed Systems. In Proceedings of the ACM SIGSOFT Workshop on Self-Managed Systems, pages 90-94. ACM Press, 2004.

[25] J. J. Li, D. M. Weiss, and H. Yee. An Automatically-Generated Run-Time Instrumenter to Reduce Coverage Testing Overhead. In Proceedings of the International Workshop on Automation of Software Test, pages 49-56. ACM Press, 2008.

[26] T. Lindholm, F. Yellin, G. Bracha, and A. Buckley. The Java $\mathbb{R}$ Virtual Machine Specification, Java SE 7 Edition. Addison-Wesley, 2013.

[27] JVM Tool Interface. Oracle Corporation, 2007. http://docs. oracle.com/javase/7/docs/platform/jvmti/jvmti.html.

[28] Java Platform, Standard Edition 8 Developer Preview Releases. Oracle Corporation, 2013. http://jdk8.java.net/download.html.

[29] C. Pavlopoulou and M. Young. Residual Test Coverage Monitoring. In Proceedings of the International Conference on Software Engineering, pages 277-284. ACM Press, 1999.

[30] The SPECjvm2008 Benchmarks. Standard Performance Evaluation Corporation, 2008. http://www.spec.org/jvm2008/.

[31] M. M. Tikir and J. K. Hollingsworth. Efficient Instrumentation for Code Coverage Testing. In Proceedings of the ACM SIGSOFT International Symposium on Software Testing and Analysis, pages 86-96. ACM Press, 2002.

[32] C. Wimmer and H. Mössenböck. Optimized Interval Splitting in a Linear Scan Register Allocator. In Proceedings of the ACM/USENIX International Conference on Virtual Execution Environments, pages 132-141. ACM Press, 2005. 